\title{
Aquatic insect community structure revealed by eDNA metabarcoding derives indices for environmental assessment
}

\author{
Noriko Uchida ${ }^{\text {Corresp., 1, } 2 \text {, Kengo Kubota }}{ }^{2}$, Shunsuke Aita ${ }^{3}$, So Kazama ${ }^{2}$ \\ ${ }^{1}$ International Research Institute of Disaster Science, Tohoku University, Sendai, Miyagi, Japan \\ 2 Department of Civil and Environmental Engineering, Tohoku University, Sendai, Miyagi, Japan \\ 3 School of Engineering, Tohoku University, Sendai, Miyagi, Japan \\ Corresponding Author: Noriko Uchida \\ Email address: noriko.uchida.d1@tohoku.ac.jp
}

Environmental DNA (eDNA) analysis provides an efficient and objective approach for monitoring and assessing ecological status; however, studies on the eDNA of aquatic insects, such as Ephemeroptera, Plecoptera, and Trichoptera (EPT), are limited despite its potential as a useful indicator of river health. Here, we investigated the community structures of aquatic insects using eDNA and evaluated the applicability of eDNA data for calculating assessment indices. Field surveys were conducted to sample river water for eDNA at six locations from upstream to downstream of two rivers in Japan in July and November 2016. Simultaneously, aquatic insects were collected using the traditional Surber net survey method. The communities of aquatic insects were revealed using eDNA by targeting the cytochrome oxidase subunit I gene in mitochondrial DNA via metabarcoding analyses. The eDNA results revealed 63 families and 75 genera of aquatic insects, which was double than that detected by the Surber net survey (especially for families in Diptera and Hemiptera). The seasonal differences of communities were discriminated by the eDNA and Surber net survey data. Furthermore, environmental assessment indices (i.e., EPT index and Chironomidae index) calculated using the richness of operational taxonomic units at the genus level showed positive correlations with total nitrogen concentration as a surrogate parameter of the degree of organic pollution but the index calculated using the Surber net survey data did not. Our results demonstrated that eDNA analysis with higher taxonomic resolution can serve as a more sensitive environmental assessment index than the traditional method that requires biotic samples. 
1 Aquatic insect community structure revealed by eDNA

2 metabarcoding derives indices for environmental

3 assessment

4

5

6

7

8

\section{Noriko Uchida ${ }^{1,2}$, Kengo Kubota ${ }^{2}$, Shunsuke Aita $^{3}$, So Kazama ${ }^{2}$}

${ }^{1}$ International Research Institute of Disaster Science, Tohoku University, Sendai, Miyagi, JAPAN

${ }^{2}$ Department of Civil and Environmental Engineering, Tohoku University, Sendai, Miyagi, JAPAN

${ }^{3}$ School of Engineering, Tohoku University, Sendai, Miyagi, JAPAN

Corresponding Author:

Noriko Uchida ${ }^{1}$ 6-6-06, Aza-Aoba, Aramaki, Aoba-ku, Sendai, Miyagi, 980-8579, Japan

Email address: noriko.uchida.d1@tohoku.ac.jp

\section{Abstract}

Environmental DNA (eDNA) analysis provides an efficient and objective approach for monitoring and assessing ecological status; however, studies on the eDNA of aquatic insects, such as Ephemeroptera, Plecoptera, and Trichoptera (EPT), are limited despite its potential as a useful indicator of river health. Here, we investigated the community structures of aquatic insects using eDNA and evaluated the applicability of eDNA data for calculating assessment indices. Field surveys were conducted to sample river water for eDNA at six locations from upstream to downstream of two rivers in Japan in July and November 2016. Simultaneously, aquatic insects were collected using the traditional Surber net survey method. The communities of aquatic insects were revealed using eDNA by targeting the cytochrome oxidase subunit I gene in mitochondrial DNA via metabarcoding analyses. The eDNA results revealed 63 families and 75 genera of aquatic insects, which was double than that detected by the Surber net survey (especially for families in Diptera and Hemiptera). The seasonal differences of communities were discriminated by the eDNA and Surber net survey data. Furthermore, environmental assessment indices (i.e., EPT index and Chironomidae index) calculated using the richness of operational taxonomic units at the genus level showed positive correlations with total nitrogen concentration as a surrogate parameter of the degree of organic pollution but the index calculated using the Surber net survey data did not. Our results demonstrated that eDNA analysis with higher taxonomic resolution can serve as a more sensitive environmental assessment index than the traditional method that requires biotic samples. 
40

41

42

43

44

45

46

47

48

49

50

51

52

53

54

55

56

57

58

59

60

61

62

63

64

65

66

67

68

69

70

71

72

73

74

75

76

77

78

79

\section{Introduction}

Stream ecosystems are threatened by global climate changes and anthropogenic impacts, including damming, water abstraction, and land-use changes (WWF, 2016). For sustainable development and resource use of freshwater, there is a need to effectively manage stream environments, which requires effective methods and indicators to measure and assess environmental impacts. Aquatic insects are commonly used as indicators of environmental health due to their high sensitivity to deterioration of water quality. They form a core component of the ecological food web in river ecosystems by feeding on producers and being preyed upon by higher consumers. Thus, monitoring of aquatic insect fauna is an effective method for assessing the environmental and ecological status. However, traditional survey methods such as kick net and Surber net sampling are subject to limitations. First, traditional field sampling processes result in data bias because the success and quality of a survey depend on the ability and skills of investigators and the accessibility of sampling sites. In addition, direct sampling methods inherently involve damage to natural habitat and organism. Second, the subsequent process of sorting and morphological identification is time-consuming and requires expertise in taxonomic identification (Baird and Hajibabaei, 2012; Haase et al., 2006). These limitations have created problems in performing high-frequency and long-term biological monitoring.

The use of environmental DNA (eDNA) is a novel biological monitoring method that can be used to overcome these limitations. Due to the simple sampling method involved (i.e., grab sampling of water, soil, etc.), eDNA monitoring can reduce the sampling bias caused by individual investigators (Rees et al., 2014; Smart et al., 2015). Moreover, it can minimize the bias associated with accessing the site because water and suspended materials, including eDNA, are transported and mixed through different environments. Therefore, eDNA can detect not only lotic animals but also lentic ones (Fernández et al., 2018; Macher et al., 2018, Deiner et al. 2016). This sampling method can also overcome ethical issues such as habitat disturbance and animal sacrifice associated with field sampling because it requires only nonbiotic samples. Furthermore, DNA-based identification can quickly provide results with higher taxonomic resolution than morphological identification (Carew et al., 2013; Elbrecht and Leese, 2015; Hajibabaei et al., 2011; Serrana et al., 2018). In addition, the necessary skills to analyze DNA can be acquired over a shorter time than those required for morphological identification. Although the characteristics of eDNA remain obscure (e.g., production and degradation rates and transportation dynamics), it can provide ecological information that is unobtainable via traditional survey methods. For example, eDNA can be used to evaluate the diversity of animals across almost the entire fauna (fish fauna: Ushio et al., 2018) or across multiple phyla (phylum in eukaryotes: Deiner et al., 2016).

Aquatic insects such as Ephemeroptera, Plecoptera, Trichoptera (the abbreviated name of these groups in combination is EPT), and Diptera have been gradually targeted in eDNA studies in pursuit of effective river management (Bista et al., 2017; Fernandes et al., 2018; Fernández et al., 2018; Hajibabaei et al., 2019a; Macher et al., 2018; Mächler et al., 2019). However, there is a 
80

81

82

83

84

85

86

87

88

89

90

91

92

93

94

95

96

97

98

99

100

101

102

103

104

105

106

107

108

109

110

111

112

113

114

115

116

117

118

119

lack of information regarding the spatial and temporal differences of community structures of aquatic insects revealed by eDNA (Bush et al., 2019; Roussel et al., 2015). Furthermore, studies examining the possible use of eDNA data for calculating environmental assessment indices and focusing on aquatic insects in stream ecosystems in a Japanese context are warranted.

Therefore, the present study aimed to evaluate the applicability of eDNA in environmental assessments. First, we revealed the community structures of aquatic insects using eDNA and the traditional Surber net survey. Subsequently, we investigated whether eDNA data and the Surber net survey data can discern the spatial and temporal differences of aquatic insect communities. We also evaluated the relationships between water quality parameters and assessment indices derived from each method.

\section{Materials \& Methods}

\section{1 eDNA sampling, filtration, and DNA extraction}

Field samplings were conducted at Hirose River and Natori River located at the Natori River basin, Miyagi Prefecture, northeast Japan. The length of the channel of Hirose River is $45.2 \mathrm{~km}$ and its catchment area is $315.9 \mathrm{~km}^{2}$. Natori River is $55.0 \mathrm{~km}$ long and its catchment area is 623.0 $\mathrm{km}^{2}$ (not including the Hirose River Basin). Sampling was conducted in July and November 2016 at six locations from upland to lowland regions along the two rivers (sites H1-H3 and N1N3; Figure 1 and Table S1). These are temperate rivers that originate in the mountains and flow through the hills at their middle reaches and through urbanized flatlands at their lower reaches and finally flow into the Pacific Ocean.

Water samples for eDNA analysis were collected at the same sites and on the same days. The plastic bottles for eDNA sampling were sterilized with $10 \%$ chlorine bleach (Kao Corporation, Tokyo, Japan), rinsed with tap water in the laboratory, and washed thrice with river water at the collection site before sampling. At each site, flowing surface water was collected and transported to the laboratory on ice in a cool box. Water samples were filtered on the same day using vacuum filtration with $47-\mathrm{mm}$ diameter glass fiber filters with a $0.7-\mu \mathrm{m}$ pore size $(\mathrm{GF} / \mathrm{F}$, Whatman, $1 \mathrm{~L} /$ filter, referring to Mächler et al., 2016). These filtered samples were stored at $-20^{\circ} \mathrm{C}$ until DNA extraction. DNA was extracted from the filters through lysis using proteinase $\mathrm{K}$ at $56^{\circ} \mathrm{C}$ for $30 \mathrm{~min}$. Then, the supernatant obtained was subjected to phenol-chloroformisoamyl alcohol extraction and ethanol precipitation. Eventually, the elution was purified using the OneStep PCR Inhibitor Removal Kit (Zymo Research, Irvine, CA, USA) with a final volume of $100 \mu 1$.

\subsection{Library preparation and sequencing}

The cytochrome oxidase subunit I (COI) gene region in mitochondrial DNA was amplified from extracted DNA using the universal primer for invertebrates developed by Folmer et al. (1994). The primer set of LCO1490 (5'-GGT CAA ATC ATA AAG ATA TTG G-3') as the forward 
120

121

122

123

124

125

126

127

128

129

130

131

132

133

134

135

136

137

138

139

140

141

142

143

144

145

146

147

148

149

150

151

152

153

154

155

156

157

158

159

primer and HCO2198 (5'-TAA ACT TCA GGG TGA CCA AAA AAT CA-3') as the reverse primer resulted in an amplification of a 658-bp fragment. For library preparation, a three-step polymerase chain reaction (PCR) was conducted. The first PCR was performed in a total volume of $20 \mu \mathrm{l}$ PCR mixture comprising $10 \mu \mathrm{l}$ of Taq $^{\mathrm{TM}}$ HS Low DNA (TaKaRa, Kyoto, Japan), $0.4 \mu 1$ each of $10 \mu \mathrm{M}$ forward and reverse primers, $17.2 \mu 1$ of ultrapure water, and $2.0 \mu 1$ of template DNA. The PCR conditions were as follows: 35 cycles at $94^{\circ} \mathrm{C}$ for $5 \mathrm{~s}, 50^{\circ} \mathrm{C}$ for $30 \mathrm{~s}, 68^{\circ} \mathrm{C}$ for 10 $\mathrm{s}$; and a final extension at $68^{\circ} \mathrm{C}$ for $7 \mathrm{~min}$. The fragment size of amplicons and the concentrations were verified by electrophoresis using the Agilent 2100 Bioanalyzer DNA7500 kit (Agilent, Santa Clara, CA, USA). PCR products were purified using the Agencourt AMPure XP (Beckman Coulter, Brea, CA, USA), and the purified products were used as templates for the following PCR. The second PCR was performed using Ex Taq Hot Start Version (TaKaRa, Kyoto, Japan) to add the overhang sequences that required amplification with the Nextera XT Index Kit for Illumina MiSeq analysis. The PCR conditions were as follows: $94^{\circ} \mathrm{C}$ for $2 \mathrm{~min}$; followed by 5 cycles of $94^{\circ} \mathrm{C}$ for $30 \mathrm{~s}, 50^{\circ} \mathrm{C}$ for $30 \mathrm{~s}, 72^{\circ} \mathrm{C}$ for $30 \mathrm{~s}$; and a final extension at $72^{\circ} \mathrm{C}$ for 5 min. The amplicons were purified in the same manner as those obtained from the first PCR, and the purified products were used as templates for the next PCR. The third PCR was performed using Ex Taq Hot Start Version and Nextera XT Indice Kit v2 Set A (Illumina, San Diego, CA, USA). The PCR conditions were followed: $94^{\circ} \mathrm{C}$ for $2 \mathrm{~min}$; followed by 8 cycles of $94^{\circ} \mathrm{C}$ for $30 \mathrm{~s}, 50^{\circ} \mathrm{C}$ for $30 \mathrm{~s}, 72^{\circ} \mathrm{C}$ for $30 \mathrm{~s}$; and a final extension at $72^{\circ} \mathrm{C}$ for $5 \mathrm{~min}$. After purification, the final PCR amplicons were quantified using the Qubit dsDNA High Sensitivity Kit. The sequencing of prepared libraries was performed using MiSeq, according to the manufacturer's instructions.

\subsection{Bioinformatics}

The sequence lengths of the amplicons were $658 \mathrm{bp}$ so the forward and reverse reads in our study could not be merged when using the MiSeq Reagent Kit v3 (600 cycles). Elbrecht and Leese (2017) have demonstrated that invertebrate species could be identified at the reverse side of the COI region through an in silico PCR approach. Therefore, we conducted a subsequent analysis using the reverse side sequence. Initially, the raw sequence reads were subjected to the Trimmomatic v0.36 software to discard low-quality sequences and read sequence lengths of $<150 \mathrm{bp}$. The filtered reads were clustered into operational taxonomic units (OTUs) using QIIME (Caporaso et al., 2010), with an identity cut-off value of $97 \%$, which is a common approach for invertebrate metabarcoding analyses (Macher et al., 2018). Subsequently, OTUs with singleton sequences were removed. The most frequently occurring sequences in each OTU were extracted as representative sequences. The assignment was performed against 3,433,026 sequences retrieved from the National Center for Biotechnology Information (NCBI) database using the following search criteria: cytochrome [all fields] AND oxidase [all fields] AND mitochondrion [filter]. After the assignment, eight orders (i.e., Ephemeroptera, Plecoptera, Trichoptera, Diptera, Coleoptera, Odonata, Megaloptera, and Hemiptera) that mostly include aquatic insect species were extracted using the QIIME script "filter_taxonomy_from_table.py." Subsequently, representative sequences of the extracted OTUs were subjected to a chimera 
160

161

162

163

164

165

166

167

168

169

170

171

172

173

174

175

176

177

178

179

180

181

182

183

184

185

186

187

188

189

190

191

192

193

194

195

196

197

198

199

check. Taxonomic identification was performed using a BLAST search and the QIIME script “assign_taxonomy.py." Because the traditional environmental assessment indices (\%EPT, $\%$ Diptera, and \%Chironomidae) require at least family-level taxonomic identification, we employed two thresholds for taxonomic identification: 97\% identity for genus-level assignation and $85 \%$ identity for family-level assignation. For macroinvertebrates, a threshold of $97 \%$ is commonly used for species/genus-level assignment (Hebert et al., 2003) but may cause a loss of sequence depth. According to our investigation and using a subset of sequence data of EPT registered in NCBI, the sequence identity for the COI region of EPT was $99 \%$ at the intraspecific level, $85 \%$ at the intragenus level, $83 \%$ at the intrafamily level, and $80 \%$ at the intra-order level (see Text S1). Therefore, a BLAST assignment was conducted with a minimum identity of 97\% (assigned at the genus-level) and 85\% (assigned at least at the family-level) and a maximum evalue of $10^{-50}$ (Fernández et al., 2018). To compare the communities among samples, we subsampled the number of sequences in each sample by a uniform number. According to the smallest numbers of sequence reads in the samples, either 250 reads for the family-level analysis or 150 reads for the genus-level analysis were randomly selected (see Table S2).

\subsection{Aquatic insect sampling using a Surber net survey and measurement of environmental parameters}

After eDNA sampling but within the same day, traditional aquatic insect collection was conducted using the Surber net survey method. A Surber net of $250-\mu \mathrm{m}$ mesh size in a $30 \times 30$ $\mathrm{cm}$ quadrat at randomly selected riffle and pool habitats at each site (total collection area: 0.18 $\mathrm{m}^{2} /$ reach) was used. Collected invertebrates were placed in $99.5 \%$ ethanol and morphologically identified using a stereomicroscope (Leica MZ APO; Leica, Germany) by referring to the identification key for the aquatic insects of Japan (Kawai and Tanida, 2018). Because morphological identification was difficult for some aquatic insects, particularly Chironomidae and some Baetidae individuals, population abundance and richness were summarized at the family level.

At the same time as aquatic insect sampling, environmental parameters such as water temperature, electrical conductivity (EC; TOA-DKK CM-21P; Japan), and pH (TOA-DKK HM20P; Japan) were measured in the field. River water samples of $50 \mathrm{ml}$ were collected from each site to obtain the concentrations of total phosphorus (TP) and total nitrogen (TN) measured in our laboratory using a QuAAtro-2HR (BLTEC Corporation, Japan).

\subsection{Community structure analysis}

We assessed the dissimilarity in community structures of aquatic insects using eDNA or Surber net survey data. Community dissimilarities were calculated based on the Sørensen index (binary Bray-Curtis index) using the presence/absence of OTU data for eDNA and the detected taxa for the Surber net survey using the "vegan" package (Oksanen et al., 2019) in R ver. 3.4.0 (R core team, 2018). Using the ordination of dissimilarity, nonmetric multidimensional scaling (nMDS) was performed to visualize the similarity in community structures and the "metaMDS" function

PeerJ reviewing PDF | (2019:04:37104:3:0:NEW 17 Apr 2020) 
200 in the "vegan" package. Furthermore, the correlations between community structures and 201 environmental parameters (i.e., water temperature, EC, TN, and TP) were determined using the 202 "envfit" function in the "vegan" package.

203

204

205

206

207

208

209

210

211

212

213

214

215

216

217

218

219

220

221

222

223

224

225

226

227

228

229

230

231

232

233

234

235

236

237

238

239

\section{Results and Discussion}

\subsection{The community structure of aquatic insects revealed by eDNA analysis}

A total of 1,235,176 sequences (50,728-168,413 sequences/sample) passed the sequence quality filter (Table S2 for the detail of metabarcoding outputs). These sequences were used to create OTUs based on $97 \%$ sequence identity. As a result, 90,948 OTUs were formed. Among these, 66,176 OTUs comprised just one sequence (singletons), which were excluded from the analysis. Finally, a total of 1,169,000 sequences (47,443-161,461 sequences/sample) generating 24,773 OTUs were analyzed. After a BLAST search, we found that $8.0 \%$ of the total sequences were assigned to aquatic insects at the family level (sequence identity $\geq 85 \%$, see Materials \&

Methods 2.3) and only $4.1 \%$ of them were assigned at the genus level (sequence identity $\geq 97 \%$ ) (Table S2).

eDNA metabarcoding detected 93 families and 104 genera before subsampling, and after subsampling, 63 families and 75 genera were detected (sequence depths were 250 reads for the family-level assignment and 150 reads for the genus-level assignment; see Tables S2 and S3 for the OTU tables before subsampling). A total of 26 families were common with the Surber net survey results. Even after subsampling, the total number of taxa detected by the eDNA method was nearly double than that detected by the Surber net survey method (Table S4). Specifically, eDNA detected 27 genera of Chironomidae (Diptera); however, these genera could not be distinguished by morphological identification. In addition, eDNA detected taxa that were mostly distributed in riparian/terrestrial habitats (e.g., Culicidae; Diptera, Cicadidae; Hemiptera) and lentic habitats (Aeshnidae and Epiophlebiidae; Odonata). 
240 According to the subsampling results of eDNA, the taxa detected in both the months were 26

241 families/25 genera, those detected in July alone were 26 families/41 genera, and those detected in

242 November alone were 11 families/9 genera. Among these, we found that the three families

243 (Ephemerellidae, Chironomidae, and Simuliidae) were commonly detected among all sites in

244 both the months (see Table S5 for details of assignment results at the family level). Conversely, a

245 number of unique taxa were detected in the communities at site H1 (11 genera in July and 9

246 genera in November; see Table S6 for details of assignment results at the genus level).

247 Previous studies have reported that compared with the use of a traditional survey method, the use 248 of eDNA in lotic systems tends to enable the detection of more taxa (Macher et al., 2018); this is 249 in contrast with the case in pond systems wherein DNA transportation is very low (Hajibabaei et 250 al., 2019a). This is because DNA is transported downstream in lotic systems, which results in the 251 additional detection of upstream communities that are overlooked by traditional methods. It has 252 been reported that fish eDNA is decomposed and transported after release from organisms, with 253 a 73\% decrease in eDNA concentration within $900 \mathrm{~m}$ downstream of the source (Nukazawa et 254 al., 2018). Even 50-250 m downstream of the source, eDNA was not reported to be detected 255 when the target organisms' abundance or biomass is small (Jane et al., 2015; Pilliod et al., 2014). 256 Thus, the DNA sampled in rivers probably includes some DNA originating from abundant 257 organisms, within an approximate distance of $1 \mathrm{~km}$ upstream. While the source materials of 258 eDNA differ depending on the organism [e.g., mucus for fish (Takeuchi et al., 2019), saliva for 259 terrestrial mammals (Rodgers and Mock, 2015; Ushio et al., 2017), and exuvia for aquatic 260 arthropods (Deiner and Altermatt, 2014)], the nature of eDNA in lotic systems may exist in a 261 similar manner. The interval between sampling sites in our study was approximately $3-5 \mathrm{~km}$; 262 therefore, the eDNA contamination among samples was assumed to be negligible. In addition, Jo 263 et al. (2017) have shown that amplification of longer DNA fragments (719 bp vs. $127 \mathrm{bp}$ ) is 264 more effective in reflecting real time biological information. The length of the amplicons in the 265 present study was relatively long (658 bp); therefore, the community structures obtained using 266 eDNA were mainly based on DNA that might have been generated recently and transported from 267 a closer area. In addition, eDNA can be used to detect taxa that are usually difficult to capture 268 using the Surber net survey method in lotic systems such as terrestrial organisms (Deiner et al., 269 2016b; Mächler et al., 2014). As shown in previous studies, terrestrial and semiterrestrial taxa 270 were also detected in our samples. These results indicate that the eDNA sampled from river 271 ecosystems provides a diverse taxonomic list that differs from the traditional Surber net sampling 272 method.

273 From the results of the family-level assignment, nine families were not detected in any eDNA 274 samples but were detected with Surber net sampling, namely, two ephemeropteran (Isonychiidae 275 and Siphlonuridae), one plecopteran (Chloroperilidae), one trichopteran (Apataniidae), one 276 dipteran (Blephariceridae), and four coleopteran (Gyrinidae, Hydrophilidae, Psephenidae, and 277 Ptilodactylidae) taxa. Of these, Isonychiidae, Blephariceridae, Hydrophilidae, Psephenidae, and 278 Ptilodactylidae have mismatched sequences with the primers used in this study, which results in 279 failure of PCR primer amplification. Therefore, the primers should be modified or new primers 
280

281

282

283

284

285

286

287

288

289

290

291

292

293

294

295

296

297

298

299

300

301

302

303

304

305

306

307

308

309

310

311

312

313

314

315

316

317

318

319

should be developed to analyze these five families. Some refined primer sets for the metabarcoding of aquatic invertebrates have been developed (Elbrecht and Leese, 2017; Hajibabaei et al., 2012). Hajibabaei et al. (2019b) also suggested that the use of multiple universal primers enables coverage of a broader range of taxa.

In addition to the primer issue, the sequence identity threshold used for taxonomic identification can be another problem for eDNA analysis. To evaluate the discrepancy between the reference library and the query sequence, we investigated intraspecific, intrageneric, and intrafamilial genetic identity (Text S1). As a result, an $85 \%$ identity threshold at the family level and a $97 \%$ identity threshold at the genus level were employed for taxonomic assignment in this study. However, this threshold might not have been achieved by some species and thus gone undetected. To overcome this issue, reference sequence data should be accumulated. Geographically separated intraspecies have low sequence identity of the COI gene (Takenaka and Tojo, 2019). Therefore, the accumulation of genetic information of local aquatic insects and the construction of a database are necessary to improve the taxonomic assignment and to avoid false negative results. There is an urgent need to overcome these issues because failure to detect some specific taxonomic groups could directly affect the assessment results based on the richness of taxa.

\subsection{Relationships between communities and environmental parameters}

Community dissimilarities among all samples using the Sørensen index for eDNA analysis and Chao index for Surber net survey data were plotted on nMDS axes (Figure 2). Visually, nMDS showed monthly differences among communities (July or November). Ordination was

significantly correlated with water temperature in all three datasets (function "envfit"; eDNA at the genus level: $\mathrm{R}^{2}=0.56, \mathrm{p}=0.016$, eDNA at the family level: $\mathrm{R}^{2}=0.81, \mathrm{p}=0.001$, and Surber net survey data at the family level: $\mathrm{R}^{2}=0.57, \mathrm{p}=0.021$; Table S7). Bista et al. (2017) have shown that eDNA targeting the chironomid community (Diptera) in a lake system can distinguish seasonal differences across communities. Similarly, the present study demonstrated that eDNA analysis targeting the aquatic insect community in a river system revealed seasonal differences.

In addition, nMDS showed that the uppermost site of the Hirose River (H1) was plotted in isolation from the other sites in both months and for all three datasets (Figure 2). This was understandable because the landscape of $\mathrm{H} 1$ differs from those of the other sites; it is a mountain stream, and its inhabitants differ from those found in other middle and lower reaches. Indeed, eDNA analysis detected multiple unique taxa at $\mathrm{H} 1$ that made differences among communities (Table S5 and S6). Conversely, in the Surber net survey, the differences among communities were revealed by the absence of taxa that could be found in the other sites, rather than the presence of unique taxa at H1 (Table S4). Thus, both eDNA analysis and Surber net survey clearly illustrated differences among communities depending on landscapes (mountain stream or middle/lower reach), whereas the factors attributable to the differences among communities differed between methods.

PeerJ reviewing PDF | (2019:04:37104:3:0:NEW 17 Apr 2020) 
320 Furthermore, the ordination based on eDNA data was significantly correlated with TN 321 concentration (function "envfit"; eDNA at the genus level: $\mathrm{R}^{2}=0.50, \mathrm{p}=0.043$, eDNA at the 322 family level: $\mathrm{R}^{2}=0.51, \mathrm{p}=0.046$ ); however, no such correlation was noted for the Surber net 323 survey data. TN concentration may not only be a direct proxy for organic pollution, but also an

324

325

326

327

328

329

330

331

332

333

334

335

336

337

338

339

340

341

342

343

344

345

346

347

348

349

350

351

352

353

354

355

356

357

358

359 indirect proxy for site characteristics. That is because agricultural and urban land use increase in the lower area in our study area, so the larger the stream order, the higher the TN concentration (Table S1). Previous studies reported that eDNA can distinguish geographical changes in communities in river systems for various fauna (fungi: Matsuoka et al., 2019; macroinvertebrates: Hajibabaei et al., 2019b; Fernández et al., 2019). Similarly, the present study showed that the community differences revealed by eDNA analysis were related to the site characteristics by water quality rather than by geographical location.

\subsection{Environmental assessment indices derived from eDNA}

The relationship between the $\mathrm{TN}$ concentration and biological environmental assessment indices (\%EPT, \%Diptera, and \%Chironomidae) was examined (Figure 3) because TN could be assumed to be a chemical indicator of water pollution. The results showed that \%EPT and \%Chironomidae derived from eDNA at genus-level resolution showed sufficient effect sizes with significant rank correlation with TN (Spearman's rank correlation; \%EPT: $r=-0.59, p=$ $0.049, \%$ Chironomidae; $r=0.69, p=0.014$; Table S7). However, the eDNA and Surber net survey results at family-level resolution did not show significant correlations. These results indicate that when eDNA data are obtained at a higher taxonomic resolution, the sensitivity of biological indices to environmental factors can be improved. Conversely, the sensitivity could be impaired if biological indices are obtained using coarse taxonomic resolution.

Emilson et al. (2017) have reported that assessment indices (i.e., the EPT index and Chironomidae index) derived from DNA metabarcoding using macroinvertebrate tissue samples were highly correlated with indices derived from the morphological survey. The present study demonstrated that the indices obtained from eDNA can also be used as a new assessment method. Although the biological indices obtained from eDNA vs. the traditional method were only compared in terms of TN concentration in the present study, new environmental indicators can be developed using eDNA data by comparison with more chemical pollution indicators such as biochemical oxygen demand and chemical oxygen demand.

While our manuscript was under review, a study that detected macroinvertebrate eDNA and applied this to the environmental status assessment of a river was reported (Fernández et al., 2019). In that report, the environmental assessment score from the IBMWP index was calculated based on the presence/absence of indicator macroinvertebrates at family-level identification. The report demonstrated that eDNA data could be used for the monitoring program that they used. Conversely, the present study showed that the EPT and Chinoromidae indices calculated using OTU richness required genus-level resolution and showed a clearer response to organic pollution compared with family-level resolution. 
360

361

362

363

364

365

366

367

368

369

370

371

372

373

374

375

376

377

378

379

380

381

382

383

384

385

386

387

388

389

390

391

392

393

394

395

396

397

398

399

400

401

\section{Conclusions}

eDNA can be used to describe differences among community structures of aquatic insects in two seasons in river systems. In addition, compared with the ordination of community derived from traditional sampling methods, that derived from eDNA analysis was correlated with the degree of water pollution. EPT and Chironomidae indices at the genus level derived from eDNA analysis data showed significant correlations with TN concentration, whereas indices derived from Surber net survey and eDNA analysis data at the family level did not. Environmental assessment indices based on ecological information but not requiring biotic samples have significant advantages such as they can be applied to places where the capture of organisms is restricted. In addition, eDNA analysis can derive benefits related to sampling such as minimal sampling effort, high taxonomic resolution, and high applicability to a broad range of species. We believe that eDNA analysis is useful for monitoring the long-term trends of changes in ecological community structure associated with environmental changes such as climate change and other anthropogenic activities, and it facilitates environmental assessment with nonbiotic samples.

\section{Acknowledgements}

The authors would like to thank Mr. Kengo Watanabe (Tohoku University, Japan) and Dr.

Yasuyuki Takemura (National Institute for Environmental Studies, Japan) for their assistance on field survey and experiments. We also greatly appreciate Dr. Kei Nukazawa (University of Miyazaki, Japan) and Dr. Yasuhiro Takemon (Kyoto University, Japan) for providing meaningful comments on our paper.

\section{References}

Baird, D.J., Hajibabaei, M., 2012. Biomonitoring 2.0: a new paradigm in ecosystem assessment made possible by next-generation DNA sequencing. Mol. Ecol. 21, 2039-2044. https://doi.org/10.1111/j.1365-294X.2012.05519.x

Bista, I., Carvalho, G.R., Walsh, K., Seymour, M., Hajibabaei, M., Lallias, D., Christmas, M., Creer, S., 2017. Annual time-series analysis of aqueous eDNA reveals ecologically relevant dynamics of lake ecosystem biodiversity. Nat. Commun. 8, 14087. https://doi.org/10.1038/ncomms14087

Bush, A., Compson, Z.G., Monk, W.A., Porter, T.M., Steeves, R., Emilson, E., Gagne, N., Hajibabaei, M., Roy, M., Baird, D.J., 2019. Studying Ecosystems With DNA Metabarcoding: Lessons From Biomonitoring of Aquatic Macroinvertebrates. Front. Ecol. Evol. 7, 434. https://doi.org/10.3389/fevo.2019.00434

Caporaso, J.G., Kuczynski, J., Stombaugh, J., Bittinger, K., Bushman, F.D., Costello, E.K., Fierer, N., Peña, A.G., Peña, J.K., Gordon, J.I., Huttley, G.A., Kelley, S.T., Knights, D., Koenig, J.E., Ley, R.E., Lozupone, C.A., McDonald, D., Muegge, B.D., Pirrung, M., Reeder, J., Sevinsky, J.R., Turnbaugh, P.J., Walters, W.A., Widmann, J., Yatsunenko, T., Zaneveld, J., Knight, R., 2010. QIIME allows analysis of high-throughput community sequencing data. Nat. Methods 7, 335336. https://doi.org/10.1038/nmeth.f.303 
402

403

404

405

406

407

408

409

410

411

412

413

414

415

416

417

418

419

420

421

422

423

424

425

426

427

428

429

430

431

432

433

434

435

436

437

438

439

440

441

442

443

444

445

446

Carew, M.E., Pettigrove, V.J., Metzeling, L., Hoffmann, A.A., 2013. Environmental monitoring using next generation sequencing: rapid identification of macroinvertebrate bioindicator species. Front. Zool. 10, 45. https://doi.org/10.1186/1742-9994-10-45

Chao, A., Chiu, C.-H., 2016. Species Richness: Estimation and Comparison, in: Balakrishnan, N., Colton, T., Everitt, B., Piegorsch, W., Ruggeri, F., Teugels, J.L. (Eds.), Wiley StatsRef: Statistics Reference Online. John Wiley \& Sons, Ltd, Chichester, UK, pp. 1-26. https://doi.org/10.1002/9781118445112.stat03432.pub2

Deiner, K., Altermatt, F., 2014. Transport Distance of Invertebrate Environmental DNA in a Natural River. PLoS ONE 9, e88786. https://doi.org/10.1371/journal.pone.0088786

Deiner, K., Fronhofer, E.A., Mächler, E., Walser, J.-C., Altermatt, F., $2016 a$. Environmental DNA reveals that rivers are conveyer belts of biodiversity information. Nat. Commun. 7, 12544. https://doi.org/10.1038/ncomms12544

Deiner, K., Fronhofer, E.A., Mächler, E., Walser, J.-C., Altermatt, F., 2016b. Environmental DNA reveals that rivers are conveyer belts of biodiversity information. Nat. Commun. 7, 12544. https://doi.org/10.1038/ncomms 12544

Elbrecht, V., Leese, F., 2017. Validation and Development of COI Metabarcoding Primers for Freshwater Macroinvertebrate Bioassessment. Front. Environ. Sci. 5. https://doi.org/10.3389/fenvs.2017.00011

Elbrecht, V., Leese, F., 2015. Can DNA-Based Ecosystem Assessments Quantify Species Abundance? Testing Primer Bias and Biomass-Sequence Relationships with an Innovative Metabarcoding Protocol. PLOS ONE 10, e0130324. https://doi.org/10.1371/journal.pone.0130324

Emilson, C.E., Thompson, D.G., Venier, L.A., Porter, T.M., Swystun, T., Chartrand, D., Capell, S., Hajibabaei, M., 2017. DNA metabarcoding and morphological macroinvertebrate metrics reveal the same changes in boreal watersheds across an environmental gradient. Sci. Rep. 7. https://doi.org/10.1038/s41598-01713157-x

Fernandes, K., Heyde, M. van der, Bunce, M., Dixon, K., Harris, R.J., Wardell - Johnson, G., Nevill, P.G., 2018. DNA metabarcoding-a new approach to fauna monitoring in mine site restoration. Restor. Ecol. 26, 1098-1107. https://doi.org/10.1111/rec.12868

Fernández, S., Rodríguez, S., Martínez, J.L., Borrell, Y.J., Ardura, A., García-Vázquez, E., 2018. Evaluating freshwater macroinvertebrates from eDNA metabarcoding: A river Nalón case study. PLoS ONE 13. https://doi.org/10.1371/journal.pone.0201741

Fernández, S., Rodríguez - Martínez, S., Martínez, J.L., Garcia - Vazquez, E., Ardura, A., 2019. How can eDNA contribute in riverine macroinvertebrate assessment? A metabarcoding approach in the Nalón River (Asturias, Northern Spain). Environ. DNA 1, 385-401. https://doi.org/10.1002/edn3.40

Folmer, O., Black, M.B., Hoeh, W., Lutz, R., Vrijenhoek, R.C., 1994. DNA primers for amplification of mitochondrial cytochrome c oxidase subunit I from diverse metazoan invertebrates. Mol. Mar. Biol. Biotechnol. 3, 294-299.

Peer) reviewing PDF | (2019:04:37104:3:0:NEW 17 Apr 2020) 
447 Haase, P., Murray-Bligh, J., Lohse, S., Pauls, S., Sundermann, A., Gunn, R., Clarke, R., 448 2006. Assessing the impact of errors in sorting and identifying macroinvertebrate samples. Hydrobiologia 566, 505-521. https://doi.org/10.1007/s10750-006-00756

Hajibabaei, M., Porter, T.M., Robinson, C.V., Baird, D.J., Shokralla, S., Wright, M.T.G., 2019a. Watered-down biodiversity? A comparison of metabarcoding results from DNA extracted from matched water and bulk tissue biomonitoring samples. PLOS ONE 14, e0225409. https://doi.org/10.1371/journal.pone.0225409

Hajibabaei, M., Porter, T.M., Wright, M., Rudar, J., 2019b. COI metabarcoding primer choice affects richness and recovery of indicator taxa in freshwater systems. PLOS ONE 14, e0220953. https://doi.org/10.1371/journal.pone.0220953

Hajibabaei, M., Shokralla, S., Zhou, X., Singer, G.A.C., Baird, D.J., 2011. Environmental Barcoding: A Next-Generation Sequencing Approach for Biomonitoring Applications Using River Benthos. PLOS ONE 6, e17497. https://doi.org/10.1371/journal.pone.0017497

Hajibabaei, M., Spall, J.L., Shokralla, S., van Konynenburg, S., 2012. Assessing biodiversity of a freshwater benthic macroinvertebrate community through nondestructive environmental barcoding of DNA from preservative ethanol. BMC Ecol. 12, 28. https://doi.org/10.1186/1472-6785-12-28

Hebert, P.D.N., Ratnasingham, S., deWaard, J.R., 2003. Barcoding animal life: cytochrome c oxidase subunit 1 divergences among closely related species. Proc. R. Soc. B Biol. Sci. 270, S96-S99. https://doi.org/10.1098/rsbl.2003.0025 Jane, S.F., Wilcox, T.M., McKelvey, K.S., Young, M.K., Schwartz, M.K., Lowe, W.H., Letcher, B.H., Whiteley, A.R., 2015. Distance, flow and PCR inhibition: eDNA dynamics in two headwater streams. Mol. Ecol. Resour. 15, 216-227. https://doi.org/10.1111/1755-0998.12285

Jo, T., Murakami, H., Masuda, R., Sakata, M.K., Yamamoto, S., Minamoto, T., 2017. Rapid degradation of longer DNA fragments enables the improved estimation of distribution and biomass using environmental DNA. Mol. Ecol. Resour. 17, e25e33. https://doi.org/10.1111/1755-0998.12685

Kawai, T., Tanida, K., 2018. Aquatic Insects of Japan: Manual with Keys and Illustrations [Japanese], The second edition. ed. Tokai University Press, Hiratsuka, Kanagawa, Japan.

Macher, J.-N., Vivancos, A., Piggott, J.J., Centeno, F.C., Matthaei, C.D., Leese, F., 2018. Comparison of environmental DNA and bulk-sample metabarcoding using highly degenerate cytochrome c oxidase I primers. Mol. Ecol. Resour. 18, 14561468. https://doi.org/10.1111/1755-0998.12940

Mächler, E., Deiner, K., Spahn, F., Altermatt, F., 2016. Fishing in the Water: Effect of Sampled Water Volume on Environmental DNA-Based Detection of Macroinvertebrates. Environ. Sci. Technol. 50, 305-312. https://doi.org/10.1021/acs.est.5b04188

Mächler, E., Deiner, K., Steinmann, P., Altermatt, F., 2014. Utility of environmental DNA for monitoring rare and indicator macroinvertebrate species. Freshw. Sci. 33, 1174-1183. https://doi.org/10.1086/678128

Mächler, E., Little, C.J., Wüthrich, R., Alther, R., Fronhofer, E.A., Gounand, I., Harvey, E., Hürlemann, S., Walser, J.-C., Altermatt, F., 2019. Assessing different 
493

494

495

496

497

498

499

500

501

502

503

504

505

506

507

508

509

510

511

512

513

514

515

516

517

518

519

520

521

522

523

524

525

526

527

528

529

530

531

532

533

534

535

536

537

538

components of diversity across a river network using eDNA. Environ. DNA 1, 290-301. https://doi.org/10.1002/edn3.33

Matsuoka, S., Sugiyama, Y., Sato, H., Katano, I., Harada, K., Doi, H., 2019. Spatial structure of fungal DNA assemblages revealed with eDNA metabarcoding in a forest river network in western Japan. Metabarcoding Metagenomics 3, e36335. https://doi.org/10.3897/mbmg.3.36335

Nukazawa, K., Hamasuna, Y., Suzuki, Y., 2018. Simulating the Advection and Degradation of the Environmental DNA of Common Carp along a River. Environ. Sci. Technol. 52, 10562-10570. https://doi.org/10.1021/acs.est.8b02293

Oksanen, J., Blanchet, F.G., Friendly, M., Kindt, R., Legendre, P., McGlinn, D., Minchin, P.R., O'Hara, R.B., Simpson, G.L., Solymos, P., Stevens, M.H.H., Szoecs, E., Wagner, H., 2019. vegan: Community Ecology Package.

Pilliod, D.S., Goldberg, C.S., Arkle, R.S., Waits, L.P., 2014. Factors influencing detection of eDNA from a stream-dwelling amphibian. Mol. Ecol. Resour. 14, 109-116. https://doi.org/10.1111/1755-0998.12159

$\mathrm{R}$ core team, 2018. $\mathrm{R}$ : A language and environment for statistical computing. $\mathrm{R}$ Foundation for Statistical Computing, Vienna, Austria.

Rees, H.C., Maddison, B.C., Middleditch, D.J., Patmore, J.R.M., Gough, K.C., 2014. REVIEW: The detection of aquatic animal species using environmental DNA - a review of eDNA as a survey tool in ecology. J. Appl. Ecol. 51, 1450-1459. https://doi.org/10.1111/1365-2664.12306

Reynoldson, T.B., Metcalfe-Smith, J.L., 1992. An overview of the assessment of aquatic ecosystem health using benthic invertebrates. J. Aquat. Ecosyst. Health 1, 295308. https://doi.org/10.1007/BF00044171

Rodgers, T.W., Mock, K.E., 2015. Drinking water as a source of environmental DNA for the detection of terrestrial wildlife species. Conserv. Genet. Resour. 7, 693-696. https://doi.org/10.1007/s12686-015-0478-7

Roussel, J.-M., Paillisson, J.-M., Tréguier, A., Petit, E., 2015. The downside of eDNA as a survey tool in water bodies. J. Appl. Ecol. 52, 823-826. https://doi.org/10.1111/1365-2664.12428

Serrana, J.M., Yaegashi, S., Kondoh, S., Li, B., Robinson, C.T., Watanabe, K., 2018. Ecological influence of sediment bypass tunnels on macroinvertebrates in damfragmented rivers by DNA metabarcoding. Sci. Rep. 8, 10185. https://doi.org/10.1038/s41598-018-28624-2

Smart, A.S., Tingley, R., Weeks, A.R., van Rooyen, A.R., McCarthy, M.A., 2015. Environmental DNA sampling is more sensitive than a traditional survey technique for detecting an aquatic invader. Ecol. Appl. 25, 1944-1952. https://doi.org/10.1890/14-1751.1

Takenaka, M., Tojo, K., 2019. Ancient origin of a dipteromimid mayfly family endemic to the Japanese Islands and its genetic differentiation across tectonic faults. Biol. J. Linn. Soc. 126, 555-573. https://doi.org/10.1093/biolinnean/bly192

Takeuchi, A., lijima, T., Kakuzen, W., Watanabe, S., Yamada, Y., Okamura, A., Horie, N., Mikawa, N., Miller, M.J., Kojima, T., Tsukamoto, K., 2019. Release of eDNA by different life history stages and during spawning activities of laboratory-reared Japanese eels for interpretation of oceanic survey data. Sci. Rep. 9, 6074. https://doi.org/10.1038/s41598-019-42641-9 
539

540

541

542

543

544

545

546

547

548

549

550
Ushio, M., Fukuda, H., Inoue, T., Makoto, K., Kishida, O., Sato, K., Murata, K., Nikaido, M., Sado, T., Sato, Y., Takeshita, M., Iwasaki, W., Yamanaka, H., Kondoh, M., Miya, M., 2017. Environmental DNA enables detection of terrestrial mammals from forest pond water. Mol. Ecol. Resour. 17, e63-e75. https://doi.org/10.1111/1755-0998.12690

Ushio, M., Hsieh, C., Masuda, R., Deyle, E.R., Ye, H., Chang, C.-W., Sugihara, G., Kondoh, M., 2018. Fluctuating interaction network and time-varying stability of a natural fish community. Nature 554, 360-363. https://doi.org/10.1038/nature25504

WWF, 2016. Living Planet Report 2016: Risk and Resilience in a New Era. WWW International, Gland, Switzerland. 
Figure 1

Study area

Sampling sites in Hirose River (from the upmost site, $\mathrm{H1}, \mathrm{H} 2, \mathrm{H} 3$; shown as orange circles) and in Natori River (N1, N2, N3; shown as blue circles) in northeast Japan. This map was modified using a digital map provided by the Geospatial Information Authority of Japan.

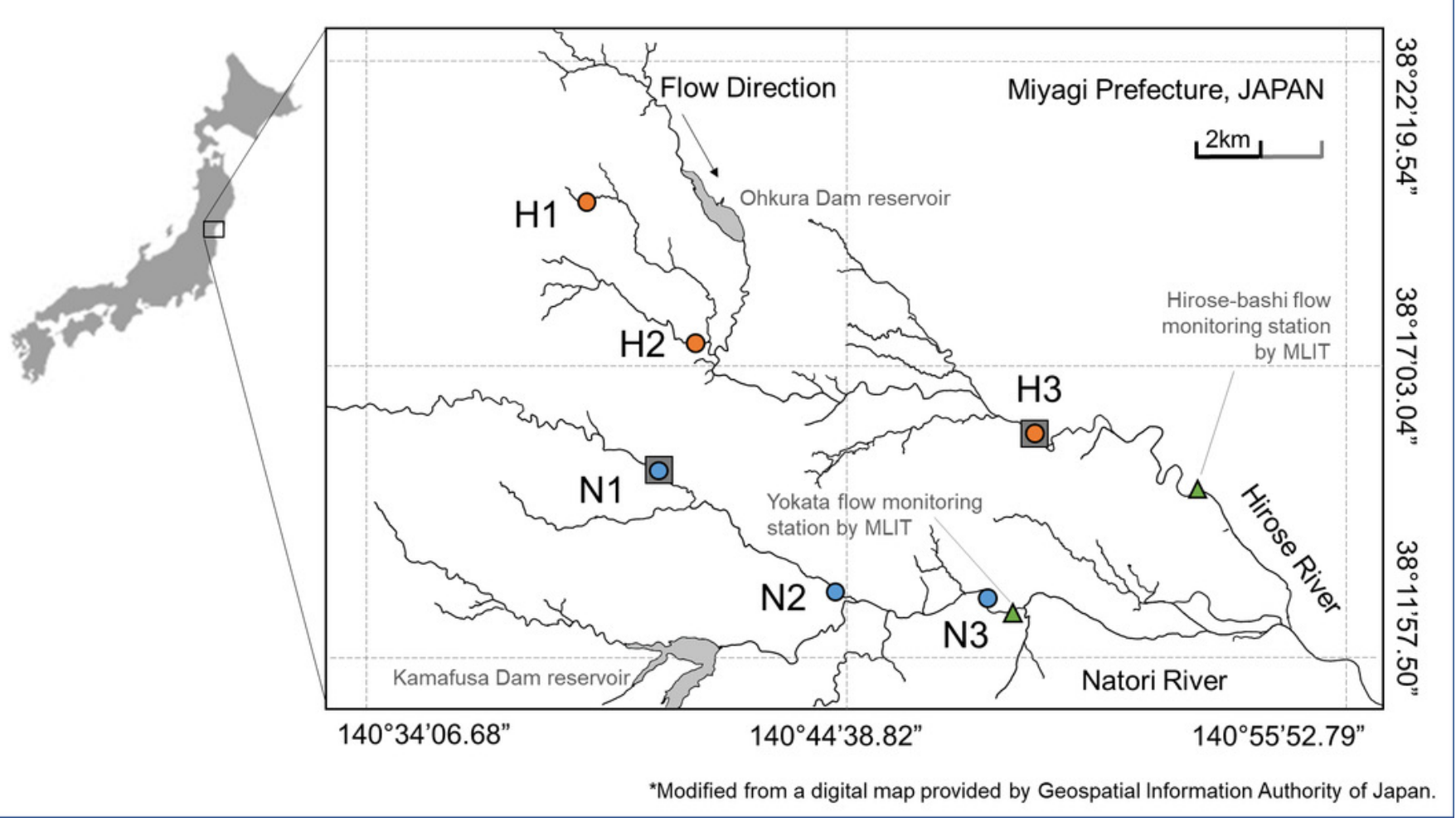




\section{Figure 2}

Non-metric multidimensional scaling (NMDS) using Sørensen dissimilarity index (P/A data).

Each panel shows the communities derived from (A) eDNA at genus level identification, (B) eDNA at family level identification, (C) Surber net (at family level identification). eDNA data were based on OTU richness with subsampled by 150 reads depth for genus level and 250 reads depth for family level. The same month is enclosed by ellipses (orange: July, blue: November). Site name and month (j: July, n: November) are displayed. The environmental parameters are shown by arrows (solid: $R^{2}>0.5$ with $p$-value $<0.05$, dotted: $R^{2} \leqq 0.5$ with $p$ value $\geqq 0.05$ ). The length of the arrow is proportional to the correlation between parameters and the community ordination.

(A)

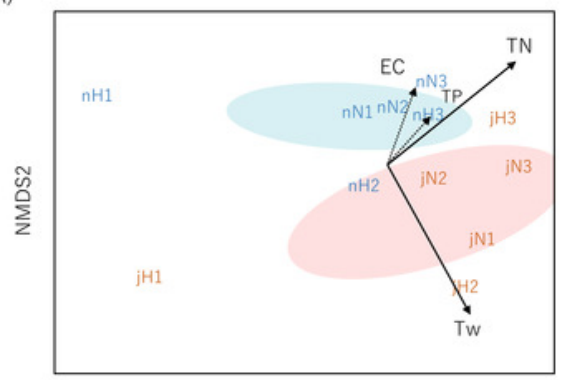

NMDS1
(B)

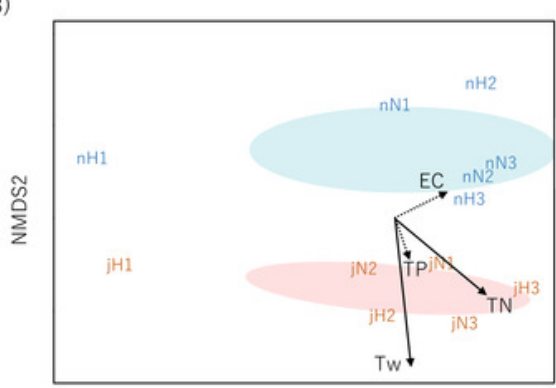

NMDS1
(C)

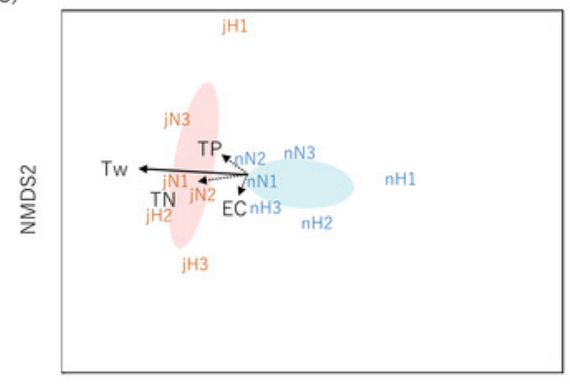

NMDS1 


\section{Figure 3}

Relationships between biological assessment indices and TN concentration.

The first (panels A-C), second (D-F), and third (G-I) rows show \%EPT, \%Diptera, and $\%$ Chironomidae, respectively. The first, second, and third columns show results based on eDNA at genus level identification, eDNA at family level identification, and Surber net survey, respectively. The indices are calculated using OTU richness data for eDNA (subsampled) and abundance data for Surber net data. Seasonal differences are represented by colors (orange: July, blue: November) and river differences are represented by plot styles (circle: Hirose River, triangle: Natori River), respectively.

\section{eDNA at genus-level}

(A)

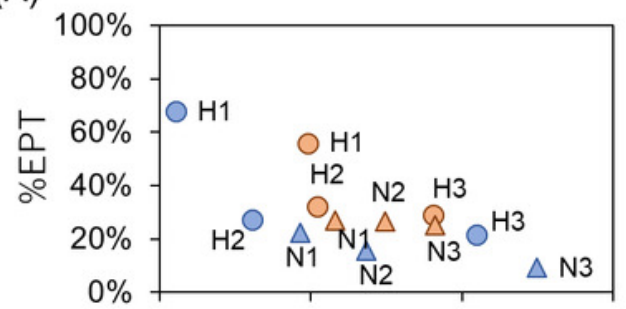

(D)

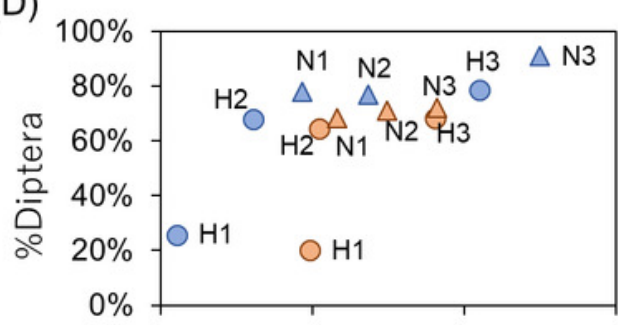

(G)

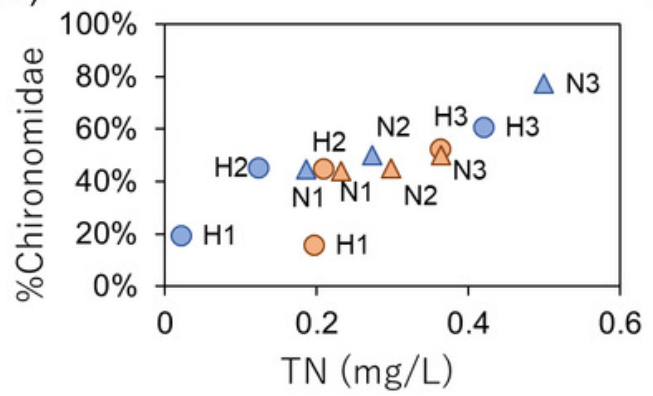

eDNA at family-level

(B)

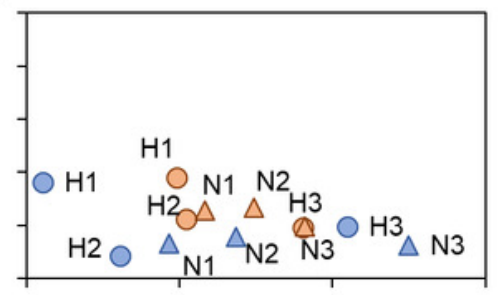

(E)

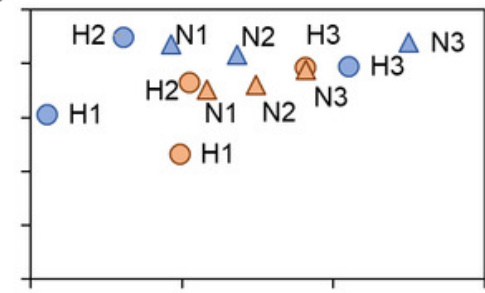

(H)

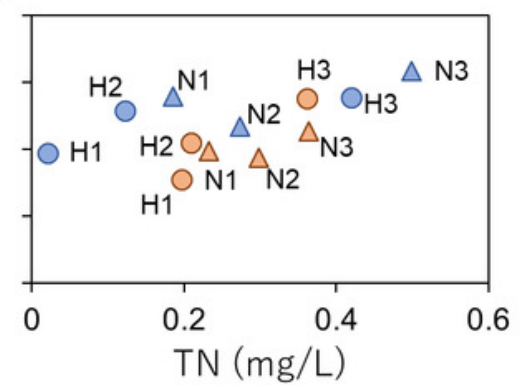

Surber net survey

(C)

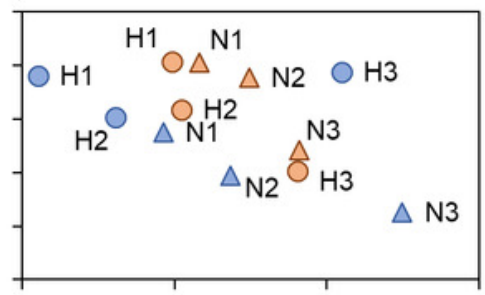

(F)

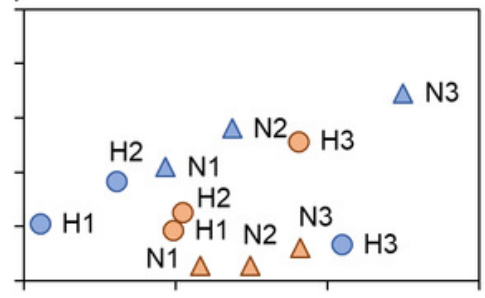

( 1 )

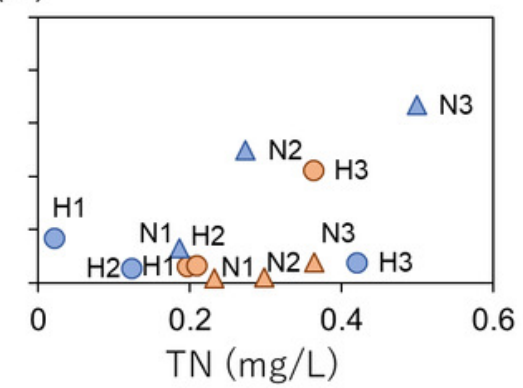

